\title{
Regra de Taylor e a conduta de Política Monetária no Brasil (1999-2006): a lição para 2007
}

\author{
Luciano D'Agostini \\ Lucas Lautert Dezordi ${ }^{* *}$
}

Em resposta a mudanças de variáveis como inflação e produto, a função de reação de política monetária tem papel importante para prever mudanças no principal instrumento de política monetária dos principais Bancos Centrais do mundo, nomeadamente a taxas de juros de curto prazo. Neste sentido, a técnica de Vetores Auto-Regressivos (VARs) é amplamente utilizada para calcular funções de reação. Como exemplos, podemos citar estudos de Bernanke-Blinder (1992), Rudebusch (1998) e Gomes-Holland (2003), este último para o Brasil.

Segundo Judd-Rudebusch (1998), ao estimar funções de reação, os pesquisadores não foram bem sucedidos para prover uma representação definitiva de comportamento das autoridades monetárias (AM) em períodos passados. Segundo eles, um fator importante pode ser mudanças na composição de especialistas dos Bancos Centrais. Tais mudanças destes especialistas podem trazer à AM preferências e concepções diferentes da tomada de decisão apropriada de política monetária. Outro fator a destacar é a influência de eventos políticos exógenos a função de reação como uma mudança de Presidente da República e de partido diferente, ou do presidente de um Banco Central, colocado como um dos mais importantes e identificáveis mudanças de comportamento de condutas de política monetária de Bancos Centrais.

Considerando duas sub-amostras delineadas por mandatos dos Presidentes da República e, baseado na Regra de Taylor, partindo da implementação do Regime de Metas de Inflação no Brasil, em junho de 1999, os objetivos do artigo são: (i) mostrar o comportamento do hiato do produto e comparar com o pib efetivo; (ii) comparar o comportamento da inflação, juros, taxa de crescimento do produto efetivo e potencial do primeiro mandato de Luís Inácio Lula da Silva (2003-2006) com o segundo mandato do presidente Fernando Henrique Cardoso $(1999-2002)^{15}$ e; (iii) calcular uma regra para taxa de juros.

Como principais resultados: (i) as taxas de juros nominais, na média, foram iguais no mandato de FHC e Lula; (ii) o governo FHC teve uma inflação média maior e taxa real de

\footnotetext{
* Doutorando em Desenvolvimento Econômico (UFPR). Endereço eletrônico: lucianodagostini@yahoo.com.br

** Doutorando em Desenvolvimento Econômico (UFPR). Endereço eletrônico: lucasdezordi@uol.com.br

15 O período inicia-se em junho de 1999 quando o sistema de Metas de Inflação foi efetivamente implantado no Brasil.
} 
juros maior; (iii) o produto potencial no governo FHC foi maior que o produto potencial do governo Lula; (iv) encontramos uma equação para a regra da taxa de juros que explica o movimento das taxas de juros reais no período do regime de Metas e; (v) como principal resultado, a equação afirma que para o governo atingir a meta de inflação de 2007, fixada em $4,5 \%$ a.a, a taxa nominal SELIC, deve cair e ter uma média de $11,9 \%$ a.a.

\section{Metodologia e fonte de dados}

Para calcular a regra da taxa de juros, considera-se uma especificação que relaciona o equilíbrio da taxa real de juros, a meta de inflação e o hiato do produto, similar a Taylor (1993), descrito em Romer (2000, p. 500):

$$
r_{t}=a+b . \pi_{t}+c \cdot\left(y_{t}\right)
$$

Onde $r_{t}$ é taxa real de juros, que é aproximadamente igual a diferença entre taxa de juros nominal $i_{t}$ e a taxa de inflação $\pi_{t}$ observada no período t e $y_{t}$ é o hiato do produto ${ }^{16}$. A equação (1) pode ser escrita como:

$$
i_{t}-\pi_{t}=a+b . \pi_{t}+c .\left(y_{t}\right)
$$

A equação (2) tem dois elementos: o primeiro é que a taxa de juros nominal deve subir mais que um-para-um em relação a inflação, de forma que a taxa nominal de juros aumente quando a inflação sobe. O segundo termo indica que a taxa de juros nominal deve cair quando produção está abaixo da natural e subir quando a produção está acima da taxa natural ${ }^{17}$. Os parâmetros b e c mostram, respectivamente, estimativas dos pesos da inflação e produção para a regra de juros. Se $\bar{r}$ denota a taxa real de juros, que presume-se ser constante quando não há hiato do produto, ou seja, $y_{t}=\bar{y}$, a equação (1 e 2) são equivalentes a:

$$
i_{t}-\pi_{t}=\bar{r}+b\left(. \pi_{t}-\pi^{*}\right)+c .(y)
$$

Onde:

$$
\bar{r}=a+b \cdot \pi^{*}
$$

A estimação na equação (4) não pode fixar o equilíbrio real da taxa de juros $\bar{r}$ e a meta de inflação $\pi^{*}$ simultaneamente. Estas duas condições são combinadas no termo

\footnotetext{
${ }^{16}$ Onde $y_{t}=100 .(y-\bar{y}) / \bar{y}$ calculado pelo filtro Hodrick-Prescott (1997).

${ }^{17}$ Holland (2006) recomenda a incorporação de uma variável fiscal, mas optamos por usar a regra de Taylor tradicional.
} 
constante $a$ e não podem ser identificados separadamente. No entanto, se assumimos um valor particular para a taxa de inflação de equilíbrio, que seria o valor da inflação estipulado pelo regime de metas de inflação, então, pelas estimativas de $a$ e $b$ obtemos a taxa real de juros de equilíbrio, $\bar{r}$. Assumindo que o Conselho Monetário Nacional (CMN), tanto no segundo mandato de FHC como no primeiro mandato de Lula, fixa a meta de inflação com a taxa de juros corrigindo os desvio da inflação observada com relação à meta, então a taxa de juros é exógena ao modelo.

No mais, somando um erro do tipo ruído branco na equação (2), os procedimentos matemáticos e econométricos estão resumidos nas seguintes etapas: (i) dessazonalizar a série do PIB pelo método multiplicativo; (ii) calcular o produto potencial pelo filtro HodrickPrescott $^{18}$; (iii) Efetuar o teste de raiz unitária pelo ADF; (iv) calcular os parâmetros $a, b$ e variância da equação por Mínimos Quadrados Ordinários (MQO $)^{19}$.

$\mathrm{Na}$ tentativa de previsão da regra de taxa de juros usaram-se dados mensais de junho de 1999 a dezembro de 2006. Utilizou-se o IPCA por ser a taxa de inflação utilizada pelo CMN para estabelecer a Meta de Inflação anual. Para a taxa nominal de juros utilizou-se a SELIC porque esta é a taxa pelo qual o BCB tenta controlar a inflação. Para o produto utilizou-se o PIB mensal em valores correntes medida em milhões de reais.

\section{Análise dos resultados}

Através do filtro $\mathrm{HP}$, os gráficos 1,2 e 3 mostram respectivamente, o comportamento da diferença do produto, y em relação ao potencial, $\bar{y}$, a estimativa do nível de atividade econômica e a taxa de crescimento do produto potencial. O gráfico 1 mostra que valores superiores a linha de tendência indicam que o PIB efetivo está acima do potencial e vice-versa.

\footnotetext{
18 Além do filtro Hodrick-Prescott (1997), que ajustam uma tendência para a série analisada, outros métodos para cálculo do produto potencial são encontrados em Perron (1989), onde calcula uma tendência linear segmentada; Clarida-Gertler-Gali (1997) que usam uma tendência quadrática e; Taylor (1993) que usa uma tendência linear aplicando logaritmos.

${ }_{19}$ Holland (2005) estima a reação do Bacen pelo Método GMM, conforme Clarida, Gali e Gertler (1997), calculando o hiato do produto pelo método da tendência linear e pelo filtro HP. Segundo Holland (2005) foi mais expressivo e adequado usar o filtro HP para cálculo do produto potencial no regime de metas. Usaremos neste trabalho também o filtro HP.
} 
Gráfico 1. Valor efetivo e a Tendência de Longo Prazo do PIB Brasileiro pelo Filtro HP (1999-2006)

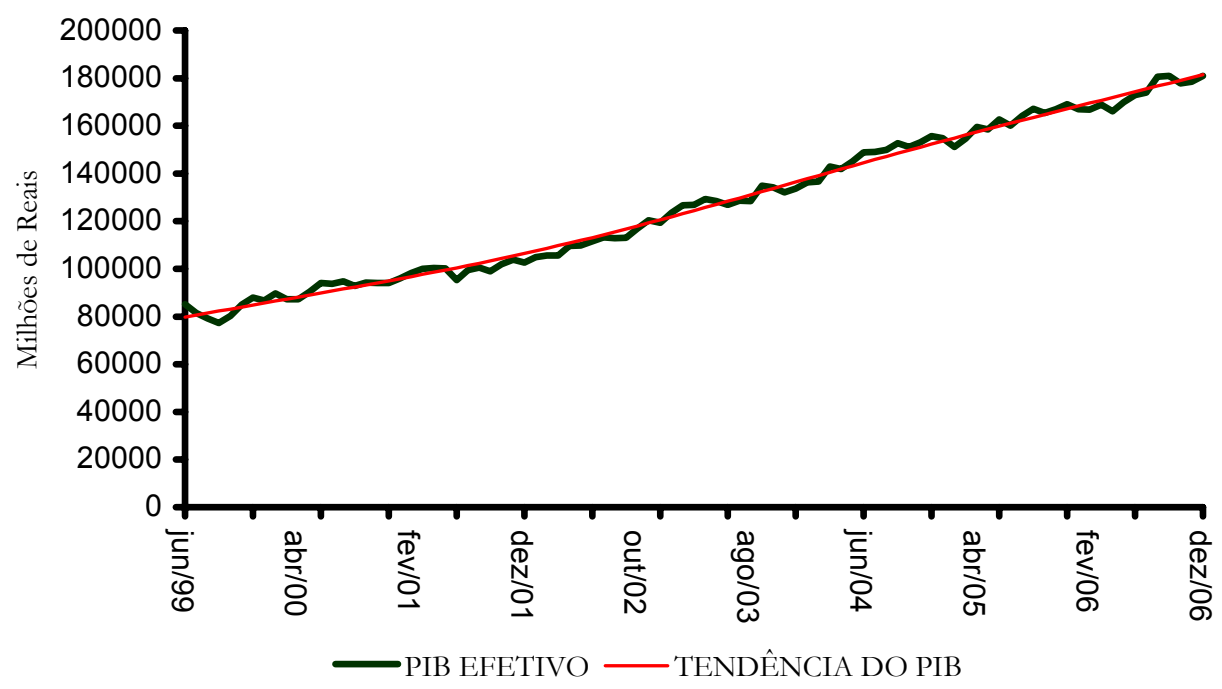

Fonte: Dados da pesquisa

Gráfico 2. Estimativa do Nível de Atividade Econômica (em \%)

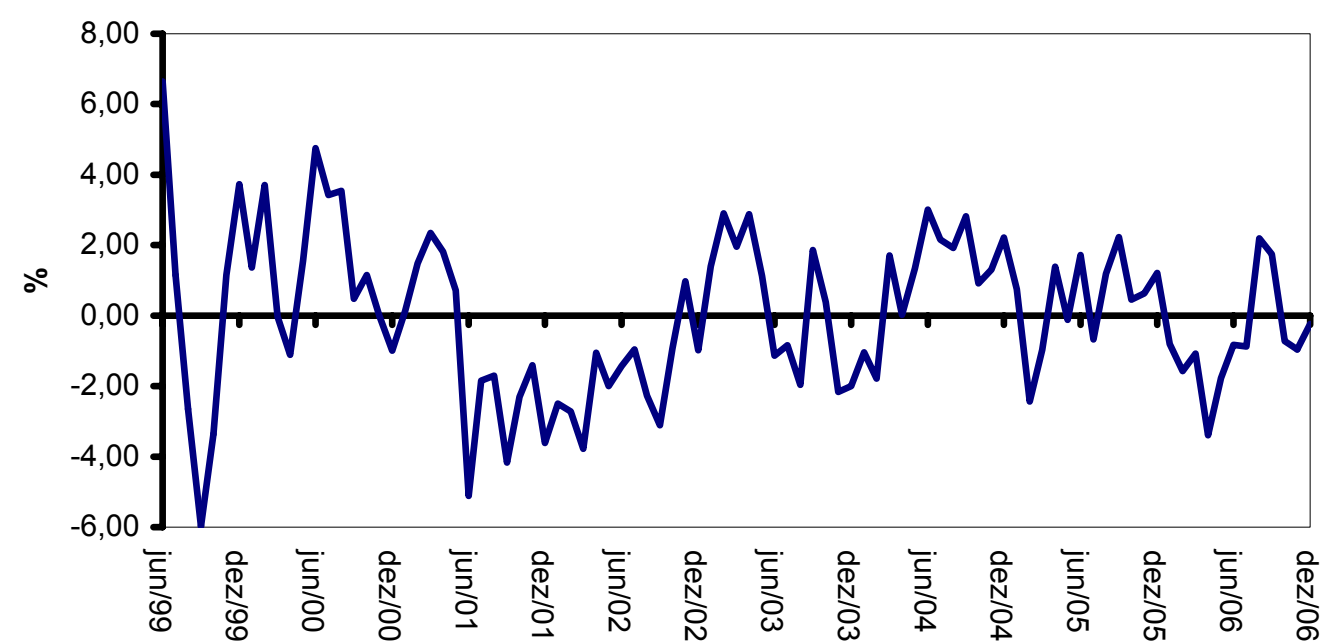

Fonte: Dados da pesquisa

Nota: calculado pelo Filtro HP (1999-2006)

O gráfico 2 mostra o resultado do nível de atividade econômica que usaremos na Regra de Taylor para estimar a taxa de juros nominal recomendada para a economia. Apesar da tendência de longo prazo apresentada no gráfico 1 ter um comportamento muito mais suave do que o PIB efetivo da economia, sua taxa de crescimento varia ao longo do tempo. 
Para ilustrar este ponto, o gráfico 3 apresenta a taxa de crescimento do produto potencial com base na tendência obtida pelo filtro HP. Como é de se esperar de uma média móvel, o crescimento potencial da economia segue a evolução do crescimento efetivo do PIB.

\section{Gráfico 3. Crescimento do Produto Potencial baseado no Filtro HP - 1999-2006 (\%)}

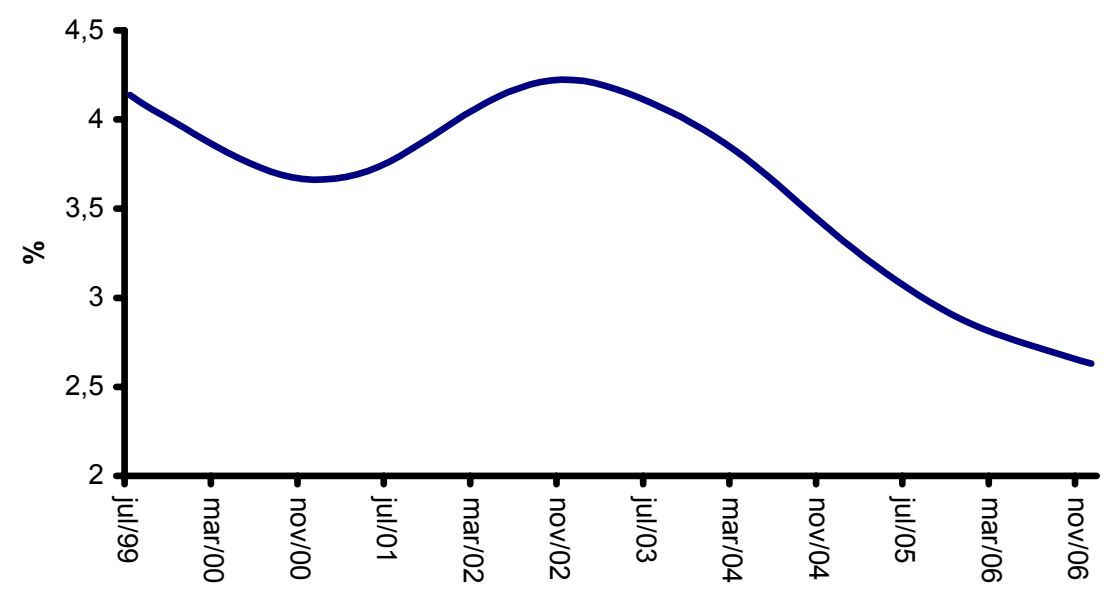

Fonte: Dados da pesquisa

Conforme o gráfico 3, no início do Regime de Metas de Inflação, compreendido no governo FHC (1999-2002), a taxa de crescimento estimada de longo prazo do produto potencial da economia brasileira foi maior que à expansão verificada de 2003-2006, período do governo Lula. Em janeiro de 2003, data da posse do governo Lula, a taxa de crescimento do produto potencial estimada estava em aproximadamente $4,2 \%$ a.a.

Desde então não há uma recuperação da estimativa da taxa de crescimento do produto potencial. Em termos quantitativos a estimativa da taxa de crescimento só declina. Ao final da amostra em dezembro de 2006 ela atinge seu valor mínimo em torno de 2,63\% a.a. A Tabela 1 mostra estimativas dos valores da taxa real de juros, da inflação e do produto potencial para o governo FHC (1999-2002) e para o governo Lula (2002-2006).

Tabela 1. Taxa de juros, Produto Potencial e Inflação (1999-2006)

\begin{tabular}{llll}
\hline Período de Metas de Inflação & $\begin{array}{c}\text { Jun/99 } \\
\text { dez/06 }\end{array}$ & $\begin{array}{c}\text { a Período } \\
\text { FHC 99/02 }\end{array}$ & $\begin{array}{l}\text { Período } \\
\text { Lula/3 jun }\end{array}$ \\
\hline Média da Taxa nominal de Juros & $18,43 \%$ & $18,34 \%$ & $18,49 \%$ \\
Média da taxa real de juros & $9,97 \%$ & $10,33 \%$ & $9,65 \%$ \\
Inflação anual média acumulada (IPCA) & $7,72 \%$ & $7,27 \%$ & $8,12 \%$ \\
Inflação Acumulada (IPCA) & $74,01 \%$ & $34,82 \%$ & $29,07 \%$ \\
Inflação do ultimo mês (IPCA) & $0,31 \%$ & $2,10 \%$ & $0,31 \%$ \\
Estimativa Média - Crescimento do PIB Potencial & $3,64 \%$ & $3,91 \%$ & $3,39 \%$ \\
\hline
\end{tabular}

Fonte: IBGE, BCB-DEPEC e do próprio autor. 
Como apresentado na tabela 1, na amostra de 1999 a 2006, o IPCA acumulado foi de 74,01\%, com média de 7,72\% a.a. Diante de uma taxa de juros nominal média de 18,43\% a.a., tivemos no período de Metas de Inflação, uma taxa de juros real média de 9,97\% a.a.

Durante o período FHC, coluna 2 da Tabela 1, o IPCA acumulado foi de 34,82\% a.a, com média de 7,27\% a.a. Diante de uma taxa de juros nominal média de 18,34\% a.a, observou-se que a taxa de juros real média foi de 10,33\% a.a.

Por fim, no primeiro mandato do Presidente Lula, o IPCA acumulado foi de 29,07\%, com média de 8,12\% a.a. Diante de uma taxa de juros nominal média de 18,49\% a.a., tivemos no período Lula uma taxa de juros real média de 9,65\% a.a.

Comparando-se os dois governos dentro do regime de Metas de Inflação observa-se que (i) tivemos uma inflação anual média acumulada pelo IPCA menor no governo Lula em relação ao FHC; (ii) uma taxa real de juros ligeiramente maior no governo FHC (10,33\% a.a) contra 9,65\% a.a no governo Lula; e (iii) mesmo com a taxa real de juros maior no período FHC tivemos a taxa média de crescimento do produto potencial estimada maior que o governo Lula (3,91\% contra 3,39\% a.a.).

Portanto, a relação entre inflação, taxa real de juros e produto no regime de Metas de Inflação no Brasil segue os fundamentos de Taylor (1993): para combater a inflação houve o custo da taxa real de juros média alta. Por sua vez, a taxa real de juros média alta fez com que o produto potencial médio nos dois governos fosse baixa, sendo no governo Lula ligeiramente menor que no governo FHC. Assim montam-se 2 cenários dentro do regime de Metas, mostrado no Quadro 1.

Quadro 1. Dois cenários no regime de metas de inflação - comparação dos governos (1999-2006)

\begin{tabular}{|l|l|l|l|l|}
\hline Governo & $\begin{array}{c}\text { Inflação } \\
\text { anual média }\end{array}$ & \multicolumn{1}{|c|}{$\begin{array}{c}\text { Inflação } \\
\text { Acumulada }\end{array}$} & $\begin{array}{c}\text { Taxa de } \\
\text { Juros Real }\end{array}$ & $\begin{array}{c}\text { PIB } \\
\text { Potencial }\end{array}$ \\
\hline FHC & Mais Baixa & Mais Alta & Mais Alta & Mais Alto \\
\hline Lula & Mais Alta & Mais Baixa & Mais Baixa & Mais Baixo \\
\hline
\end{tabular}

Fonte: Próprio autor.

No mais, o modelo estimado da Regra de Taylor é ${ }^{20}$ :

$$
\bar{r}=0,851-0,687 . \pi_{t}-0,19 . y
$$$$
(2,09) \quad(-10,68) \quad(-9,20) \quad \mathrm{DW}=1,86 \quad \mathrm{R}^{2}=0,37
$$

\footnotetext{
${ }^{20}$ Estatísticas t entre parênteses, significativos a 5\%; DW (estatística Durbin-Watson); $\mathrm{R}^{2}$ é o coeficiente de determinação.
} 
A equação estimada mostra que a taxa real de juros diminui quando a inflação e o hiato do produto aumentam. Em especial a sensibilidade da taxa de juros real em relação a mudança da inflação, dado por $\beta=-0,67$, é maior do que uma mudança no hiato do produto, dado pelo parâmetro $\lambda=-0,19$. Isto significa que a taxa de juros real varia mais devido a mudança dos níveis da inflação do que mudanças dos níveis de produto. O coeficiente de determinação, $\mathbf{R}^{2}$, para o período é de 0,37 , explicando bem as variações da taxa de juros no período considerado.

Dado a SELIC, o IPCA e o hiato do produto, o gráfico 4 mostra que a equação estimada captura com certa precisão a taxa de juros real mensal, tanto no período de FHC quanto de Lula, salvo o período de transição dos governos, nas amostras de janeiro a março de 2003. No geral, a amplitude da Regra estimada é um pouco menor que amplitude observada.

Gráfico 4. Resíduos e a Regra de Taylor Estimada comparada aos valores atuais $1999-2006$, em \% a.m.

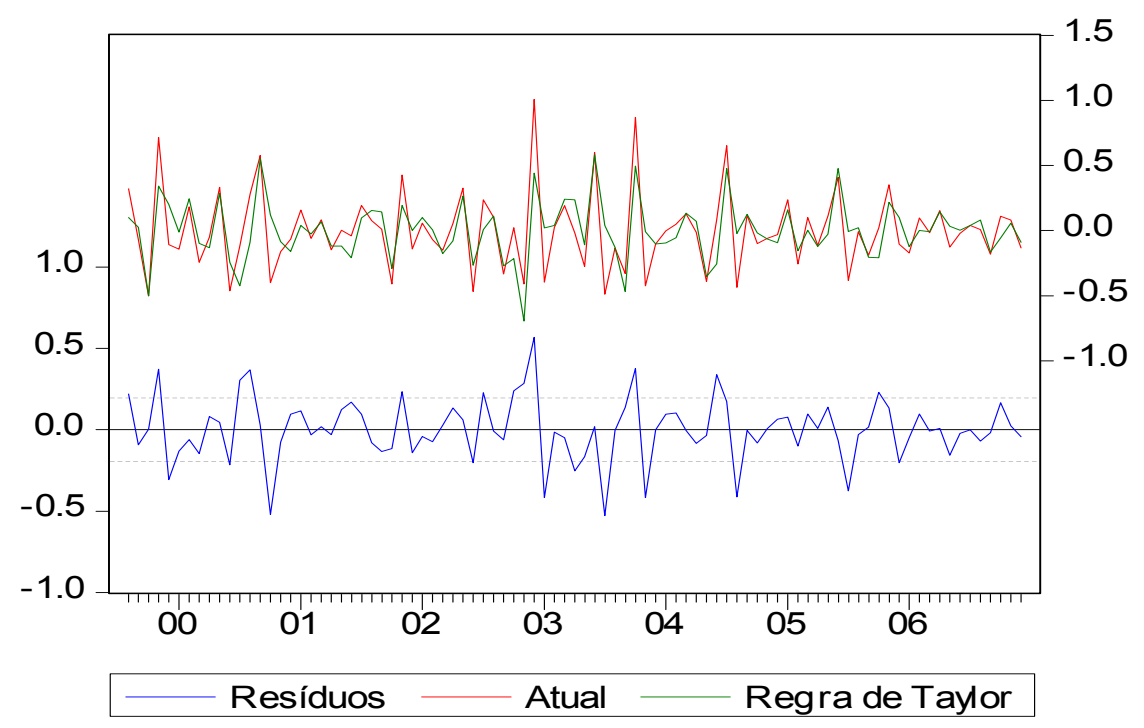

Fonte: Próprio autor.

No mais, sabe-se que no longo prazo, quando não há hiato do produto, $y_{t}=\bar{y}$, a inflação observada é igual a meta, $\pi_{t}=\pi^{*}$. Em particular, sabendo que o governo brasileiro tem uma Meta de Inflação para 2007 de 0,368\% a.m, equivalente a 4,5\% a.a, a taxa real de juros deve ser:

$$
\bar{r}=\alpha+\beta . \pi^{*} \rightarrow \bar{r}=0,850875-0,687.0,368 \rightarrow \bar{r}=0,598 \text { a.m. }
$$

O resultado de 0,598\% a.m é equivalente a aproximadamente $7,42 \%$ a.a. Como a taxa de juros nominal é a soma da taxa de juros real e inflação, o resultado mostra que para o 
Banco Central atingir a meta de inflação em 2007, a taxa nominal de juros SELIC deve cair de forma que a média deve ficar em torno de 11,9\% ao ano. Portanto o modelo estimado da Regra de Taylor, proposto por Romer (2000 p.501), indica que há espaço para queda da taxa de juros SELIC em 2007.

\section{Conclusões}

Baseado na Regra de Taylor, seguindo Romer (2000 p.500), durante o regime de Metas de Inflação, considerou-se duas sub-amostras delineadas por mandatos dos presidentes da República, nomeadamente, o segundo mandato de FHC (junho de 1999 a dezembro de 2002) e o primeiro mandato de Lula (janeiro de 2003 a dezembro de 2006).

Após efetuar a dessazonalização da série do PIB através do método multiplicativo de ajustamento sazonal; calcular o hiato do produto pelo filtro HP; calcular a SELIC mensal, com base no IPCA; e a SELIC observadas, encontramos a taxa real de juros mensal no período considerado. Então, estimamos a equação similar a Taylor, porém, diferentemente do artigo original onde usamos dados trimestrais, usamos dados mensais de juros, inflação e produto.

Como principais resultados: as taxas de juros nominais, na média foram iguais no mandato de FHC e Lula (18,43\% a.a contra 18,49\% a.a); no governo FHC observa-se uma inflação menor $(7,27 \%$ a.a. contra $8,12 \%$ a.a) e taxa real de juros ligeiramente maior $(10,33 \%$ a.a contra $9,65 \%$ a.a).

Quanto ao produto potencial, o filtro HP indicou que o produto potencial médio no governo FHC foi maior que no período Lula (3,91\% a.a contra 3,39\% a.a.). Pelo mesmo método observa-se que o produto potencial no governo Lula decresce a cada período de tempo que passa. As estimativas mostram que na data da posse do governo Lula, a taxa de crescimento do produto potencial estava em $4,2 \%$ ao ano e no fim do primeiro mandato, a taxa declinou para $2,63 \%$ a.a.

Por fim, a equação para a regra da taxa de juros explica bem o movimento das taxas de juros reais no período do regime de Metas, salvo o período de janeiro a março de 2003. Como principal resultado a equação recomenda que para atingir a meta de inflação de 2007, fixada em 4,5\% a.a, a taxa nominal SELIC deve cair e ficar com uma média de 11,9\% a.a. e a taxa real de juros em torno de 7,42\% ao ano.

Como último detalhe, portanto, sugere-se ao COPOM diminuir a taxa SELIC mais rapidamente, efetuando cortes nas próximas reuniões de 0,5 p.b. a 0,75 p.b., em vez de fazer cortes conservadores de 0,25 p.b. como temos observado nas reuniões em 2007. 


\section{Anexo}

Para garantir a estacionariedade das séries de juros, inflação e hiato do produto realizou-se o teste $\mathrm{ADF}^{21}$ para detectar a presença de raiz unitária e a ordem de integração. Com as séries em nível, o teste $\mathrm{ADF}$, a 1\% de significância, não rejeitou a hipótese de raiz unitária com qualquer equação defasada, ou seja, com tendência e intercepto, somente intercepto, ou sem tendência e intercepto). Sendo assim, o teste ADF foi refeito com as variáveis em primeira diferença, concluindo-se que para a série de juros nominal, SELIC, o teste com a equação sem intercepto e tendência e duas defasagens não rejeita a hipótese de raiz unitária a 1\% de significância (comparando com a Tabela de Mackinnon). Da mesma forma isto acontece para o IPCA e taxa real de juros, ambos com 6 defasagens e com o nível de atividade econômica com 4 defasagens. Estes resultados, indicados no Anexo 1 a 4, mostram que todas as variáveis em nível foram consideradas integradas de ordem um, I(1) e que as variáveis em primeiras diferenças foram consideradas estacionárias com ordem de integração zero, $\mathrm{I}(0)$.

Anexo 1. Teste de raiz unitária em nível e primeiras diferenças a 1\% de significância

\begin{tabular}{|c|c|c|c|c|c|}
\hline Variável & Valores $\mathrm{ADF}_{\text {calc }}$ em módulo & VC a $1 \%$ & Equação & Defasagens & i(d) \\
\hline Juros & 3,02 a $2,34<\left|{ }_{\tau}{ }_{\tau}\right|$ e 2,1 a $3,10<\left|{ }_{\mu}{ }_{\mu}\right|$ & $-4,05\left({ }_{\tau}^{\tau} \tau\right)$ e $-3,49\left({ }^{\top} \mu\right)$ & $\mathrm{I} / \mathrm{T}$ e I & 12 até 2 & $\mathrm{I}(1)$ \\
\hline Juros Reais & 1,51 a $3,02<\left|{ }_{\tau}{ }_{\tau}\right|$ e 1,1 a $3,40<\left|{ }_{\mu}{ }_{\mu}\right|$ & $-4,05\left({ }_{\tau}^{\tau}\right)$ e $-3,49\left({ }^{\tau} \mu\right)$ & $\mathrm{I} / \mathrm{T}$ e I & 12 até 2 & $\mathrm{I}(1)$ \\
\hline Preços & 0,97 a $3<\left|{ }^{\tau}{ }_{\mu}\right|$ e 0,4 a $2,28<\left.\right|^{\tau} \mid$ & $-3,52\left({ }^{\tau} \mu\right)$ e $-2,59\left(^{\tau}\right)$ & I e S T/I & 12 até 2 & $\mathrm{I}(1)$ \\
\hline Produto & 2,53 a $4,01<\left|{ }_{\tau}{ }_{\tau}\right|$ e 0,6 a $2,38<\left|{ }_{\mu}{ }_{\mu}\right|$ & $-4,05\left({ }_{\tau}^{\tau}\right)$ e $-3,4\left({ }^{\tau} \mu\right)$ & $\mathrm{I} / \mathrm{T}$ e I & 12 até 2 & $\mathrm{I}(1)$ \\
\hline$\Delta$ juros & $|-4,39|>|\tau|$ & $-2,59(\tau)$ & $\mathrm{S} \mathrm{I} / \mathrm{T}$ & 2 & $\mathrm{I}(0)$ \\
\hline$\Delta$ juros reais & $|-5,54|>\left.\right|^{\tau} \mid$ & - 2,59 (ㄱ) & $\mathrm{S} \mathrm{I} / \mathrm{T}$ & 6 & $\mathrm{I}(0)$ \\
\hline$\Delta$ preços & $|-5,29|>\left.\right|^{\tau} \mid$ & $-2,59(\tau)$ & $\mathrm{S} I / \mathrm{T}$ & 6 & $\mathrm{I}(0)$ \\
\hline$\Delta$ produto & $|-7,66|>\left.\right|^{\tau} \mid$ & $-2,59\left({ }^{(\tau)}\right.$ & $\mathrm{S} \mathrm{I} / \mathrm{T}$ & 4 & $\mathrm{I}(0)$ \\
\hline
\end{tabular}

Fonte: Dados da Pesquisa

$\mathrm{NOTA}_{1}$ : $\Delta$ Variáveis em Primeira Diferença; $\mathrm{Vc}=$ Valor Crítico; $\mathrm{I} / \mathrm{T}=$ Intercepto e Tendência; I=Intercepto; $\mathrm{S}$ $\mathrm{I} / \mathrm{T}=$ Sem Intercepto e Sem Tendência; $\mathrm{T}=$ Tendência, $\mathrm{I}(\mathrm{d})=$ Ordem de Integração

NOTA 2 : Tabela dos valores críticos(vc) $\tau_{\tau}, \tau_{\mu}$ e $\tau$ são encontrados Fuller (1976), Dickey-Fuller $(1979,1981)$.

\section{Referências bibliográficas}

Bernanke, B. S.; Blinder, A. S. The Federal Funds Rate and the Channels of Monetary Transmission. American Economic Review 82, pp. 901-921.1992.

Clarida, R.; Gali J.; Gertler, M. Monetary Policy Rules in Practice: Some International Evidence. NBER W orking Paper Series, n. 6254, novembro. 1997

D'Agostini, L. L. M. Monismo versus Não-Monismo no Brasil (1994-2004): uma Abordagem Econométrica por Vetores auto-Regressivos com Restrição. Curitiba: UFPR, 2004. 143p. Dissertação (Mestrado em Desenvolvimento Econômico).

${ }^{21}$ Ver os procedimentos metodológicos em D’Agostini (2004, p. 42-50; 89-99). 
Gomes, C.; Holland, M. Regra de Taylor e política monetária em condições de endividamento público no Brasil. Revista de Economia, n.4, n.2, jul/dez, 2003.

Holland, M. Monetary and exchange rate policy in Brazil after inflation targeting”. XXXIII Encontro Nacional de Economia, ANPEC, Natal, 2005.

Holland, M. Por que as taxas de juros reais de curto prazo são tão elevadas no Brasil? Boletim Economia \& Tecnologia, ano 02, v. 4, pp.27-42, Jan./Mar. CEPEC-UFPR, 2006.

Hodrick, R.J.; Prescott E. C. Postwar U.S. Business Cycles: An Empirical Investigation. Journal of Money, Credit, and Banking, 29, 1-16. 1997

Judd, J.; Rudebusch, G. Taylor's Rule and the FED: 1970-1997. Federal Reserve Bank of San Francisco. Economic Review, n. 3, p.1-14. 1998.

Perron, P. "The Great Crash, the Oil Price Shock, and the Unit Root Hypothesis." Econometrica 57, pp. 1361-1401. 1989

Romer, D. Advanced macroeconomics. 2a. Ed. Mc Graw-Hill, 2000. 1995.

Rudebusch, G. Do Measures of Monetary Policy Shocks in a VAR Make Sense? International Economic Review 39(4) pp. 907-941, 1998.

Taylor, J. B. Discretion Versus Policy Rules in Practice. Carnegie-Rochester Conference Series on Public Policy 39, pp. 195-214. 1993. 